\title{
The integral form of supergravity
}

\section{Castellani, ${ }^{a, b}$ R. Catenacci ${ }^{a, c}$ and P.A. Grassi ${ }^{a, b}$}

${ }^{a}$ Dipartimento di Scienze e Innovazione Tecnologica, Università del Piemonte Orientale, Viale T. Michel, 11, 15121 Alessandria, Italy

${ }^{b}$ INFN - Sezione di Torino, via P. Giuria 1, 10125 Torino, Italy

${ }^{c}$ Gruppo Nazionale di Fisica Matematica, INdAM, P.le Aldo Moro 5, 00185 Roma, Italy

E-mail: leonardo.castellani@uniupo.it, roberto.catenacci@uniupo.it, pietro.grassi@uniupo.it

ABSTRACT: By using integral forms we derive the superspace action of $D=3, N=1$ supergravity as an integral on a supermanifold. The construction is based on target space picture changing operators, here playing the rôle of Poincaré duals to the lower-dimensional spacetime surfaces embedded into the supermanifold. We show how the group geometrical action based on the group manifold approach interpolates between the superspace and the component supergravity actions, thus providing another proof of their equivalence.

KEYwords: Supergravity Models, Superspaces, Differential and Algebraic Geometry

ARXiv EPrint: 1607.05193

Dedicated to the memory of Mario Tonin. 


\section{Contents}

1 Introduction 1

2 Superspace versus supergroup manifold 2

2.1 Superspace 2

2.2 Supergroup manifold 4

2.3 Equivalence 5

$3 \quad D=3, N=1$ supergravity in the two frameworks $\quad 6$

$\begin{array}{lll}4 & \text { The actions and their equivalence } & 9\end{array}$

5 Outlook and perspectives $\quad 12$

$\begin{array}{ll}\text { A Properties of the susy PCO } & 13\end{array}$

\section{Introduction}

Three dimensional supergravity is one of the simplest models of a consistent extension of general relativity that includes fermions and local supersymmetry. For this reason it has been revisited as a workable example in many textbooks and research papers (see for example [1] and [2-10]. For recent developments see for ex. [11]). It also provides a manageable model of superfield supergravity, with a superfield action integrated on superspace. ${ }^{1}$ That action (see [10]), supplemented by ad hoc constraints consistent with the Bianchi identities, provides an off-shell formulation of $D=3$ supergravity, local supersymmetry being realized as a diffeomorphism in the fermionic directions.

On the other hand, the construction of a $3 \mathrm{~d} N=1$ supergravity in the rheonomic (a.k.a. group manifold) approach ${ }^{2}$ provides a superfield action which yields both the correct spacetime equations of motion, and the constraints on the curvatures. The action is written as a Lagrangian 3-form integrated over a bosonic submanifold of the complete supermanifold. As discussed in [12], the same action can be written as the integral over the whole supermanifold of an integral form, using the Poincaré dual that encodes the embedding of the 3-dimensional bosonic submanifold.

At the moment, however, there is no explicit dictionary between the superfield approach and the group manifold approach.

In this paper we find a bridge between the two formalisms by a novel technique based on the integration of integral forms. As is well known, differential forms on superspace

\footnotetext{
${ }^{1}$ We distinguish between superspace and supermanifold. The former denotes a flat bosonic spacetime with additional fermionic coordinates, while the latter the full-fledged supermanifold according to [14].

${ }^{2}$ for reviews on the group manifold approach see for ex. [17-19] .
} 
cannot be integrated on a supermanifold $\mathcal{S M}^{(n \mid m)}$ (where $n$ and $m$ refer to the bosonic and fermionic dimensions, respectively) since there is no top form in the usual complex of differential forms. Indeed the fermionic 1-forms behave like commuting variables with respect to the wedge product and therefore there is no upper bound to the number of fermionic 1-forms. Nonetheless, one can extend the space of forms by including distributionlike forms (see for example [12-16]). These can be incorporated into a consistent differential calculus where top forms do exist, and can be integrated on the supermanifold.

The bridge between the superspace action of $[1,10]$ and the group-manifold formalism is provided by the group-manifold three-form Lagrangian $\mathcal{L}$, which is closed (in general $d \mathcal{L}=0$ when auxiliary fields are present [17]). Multiplied by a suitable closed Poincaré dual form (known in the string theory literature as Picture Changing Operator or PCO) it becomes an integral top form, and therefore can be integrated on the supermanifold. Choosing Poincaré duals in the same cohomology class does not change the action if the Lagrangian is closed.

In particular there is a canonical Poincaré dual that produces the standard spacetime action with auxiliary fields of $[1,10]$. Another Poincaré dual, differing from the first by a total derivative, leads to an expression for the action that coincides with the superfield action of $[1,10]$. Since the two Poincaré duals are in the same cohomology class, the two actions are equal.

Furthermore, the expression of the action written as the integral of a Lagrangian three-form times a PCO clarifies an additional issue. As recalled above, the superfield formulation of supergravity is redundant in the sense that one needs some constraints to limit the number of independent component fields. It would be advantageous to have the constraints built in directly into the action. This is achieved in the present formulation: the closure of the PCO implies exactly those constraints.

The paper is organised as follows. In section 2 we discuss the equivalence between superspace and group-manifold formulations in general terms. In section 3 we provide the basic ingredients for the superfield and the group manifold formulations of $D=3, N=1$ supergravity: the constraints, the Bianchi identities and their solutions. In section 4 we prove the equivalence between the group manifold (rheonomic) formulation, the component spacetime action and the superspace action. In section 5 we list some perspectives for future work and in the appendices we give some further details on the PCO.

\section{Superspace versus supergroup manifold}

We want to formulate $D=3 N=1$ supergravity in two frameworks, namely in the groupmanifold approach and the superspace approach. Let us first clarify what we mean by supersymmetric action in the two frameworks.

\subsection{Superspace}

First, we parametrize the superspace $\mathbb{R}^{(3 \mid 2)}$ with a set of coordinates $\left(x^{a}, \theta^{\alpha}\right)$ with $a=1,2,3$ and $\alpha=1,2$. The same set of coordinates will be also used to parametrize a local patch of a supermanifold $\mathcal{S} \mathcal{M}^{(3 \mid 2)}$. 
In the case of superspace (see for example the textbook [1]), the Lagrangian is a superfield $\mathcal{F}(x, \theta)$, a local functional of the superfields $\phi(x, \theta)$ of the theory. A superfield can be expanded in its components $\phi_{0}, \phi_{1, \alpha}, \phi_{2}$

$$
\phi(x, \theta)=\phi_{0}(x)+\phi_{1, \alpha}(x) \theta^{\alpha}+\phi_{2}(x) \frac{\theta^{2}}{2},
$$

with $\theta^{2} \equiv \epsilon_{\alpha \beta} \theta^{\alpha} \theta^{\beta}$ and the components are identified with the physical degrees of freedom. A generic superfield might also contain some auxiliary fields to complete the spectrum so that there is a match between off-shell bosonic and fermionic degrees of freedom.

The superspace action is the functional

$$
S_{\text {susy }}[\phi]=\int\left[d^{3} x d^{2} \theta\right] \mathcal{F}(x, \theta)
$$

where the symbol $\left[d^{3} x d^{2} \theta\right]$ refers to the integration variables. The integration over the $\theta$ 's is given by the Berezin integral. Varying the action under an infinitesimal deformation of the superfields $\phi$, we obtain the superfield equations of motion. In the case of supergravity, the superfields $\phi$ entering (2.2) are subject to constraints, and their variations have to be compatible with these constraints. Given (2.2), one can compute the Berezin integral by expanding the action in powers of $\theta$ and then selecting the highest term

$$
S_{\text {susy }}[\phi]=\left.\int\left[d^{3} x\right] D^{2} \mathcal{F}(x, \theta)\right|_{\theta=0}
$$

which is the component action written in terms of the physical fields. The superderivative is defined as $D_{\alpha}=\partial_{\alpha}+\left(\bar{\theta} \gamma^{a}\right)_{\alpha} \partial_{a}$ where $\left(\partial_{a}, \partial_{\alpha}\right)$ are the ordinary derivatives with respect to $\left(x^{a}, \theta^{\alpha}\right)$. In addition, $D^{2}=\epsilon^{\alpha \beta} D_{\alpha} D_{\beta}$.

The supersymmetry of the action is easily checked: since $\mathcal{F}(x, \theta)$ is a superfield, its supersymmetry variation is simply

$$
\delta \mathcal{F}(x, \theta)=\epsilon^{\alpha} Q_{\alpha} \mathcal{F}(x, \theta),
$$

$Q_{\alpha}$ being the supersymmetry generator satisfying the algebra $\left\{Q_{\alpha}, Q_{\beta}\right\}=2 i \gamma_{\alpha \beta}^{a} \partial_{a}$ where $\gamma_{\alpha \beta}^{a}$ are the Dirac matrices for $D=3$. The supersymmetry generator is defined as $Q_{\alpha}=$ $\partial_{\alpha}-\left(\bar{\theta} \gamma^{a}\right)_{\alpha} \partial_{a}$

The property (2.4) follows from the fact that $\mathcal{F}(x, \theta)$ is built out of superfields $\phi$, their derivative $\partial_{a}$ and superderivative $D_{\alpha}$ and products thereof.

In the case of rigid supersymmetry, the action is invariant because the variation of the Lagrangian is a total derivative.

In the case of local supersymmetry, one needs to impose the vanishing of $Q_{\alpha} \mathcal{F}(x, \theta)=0$.

There are several advantages in having a superspace action as in (2.2). It is the most economical and compact way to describe the complete action for all physical degrees of freedom of supergravity, it encodes all symmetries, it provides a powerful quantization technique, known as supergraph method, which minimises the amount of Feymann diagrams needed for a single scattering amplitude. The supersymmetry cancellations and the nonrenormalization theorems are mostly manifest. 
The main drawback of (2.2) is the lack of a fully geometrical interpretation, since it cannot be understood as an integral of a differential form on a manifold. The expression for the superfield action is usually dictated by scaling properties and Lorentz covariance, but it is not very intuitive and for constrained superfields it does not always exist. In that respect the group-manifold approach seems to overcome these problems.

\subsection{Supergroup manifold}

The logic of this approach is algebraic: one starts from a superalgebra, and to each generator $T_{A}$ corresponds a one-form (vielbein) field $\sigma^{A}$ on the supergroup manifold $G$. The vielbein satisfies the Cartan-Maurer equations:

$$
d \sigma^{A}+\frac{1}{2} C_{B C}^{A} \sigma^{B} \wedge \sigma^{C}=0
$$

The fields of the theory are identified with the various components of the vielbein $\sigma^{A}$, labelled by the adjoint index $A$. For the fields to become dynamical, they must be allowed to develop a nonzero curvature, that is to say the right-hand side of the Cartan-Maurer equations must be nonvanishing in general. This is achieved by considering deformations of the supergroup manifold, i.e. a "soft" supergroup manifold $\tilde{G}$.

A systematic procedure [17-19] leads to the construction of $d$-form lagrangians, whose restriction to a $d$-dimensional bosonic manifold reproduces the $d$-dimensional spacetime supergravity lagrangians. The local symmetries of the theory are the superdiffeomorphisms on $\tilde{G}$, and include the supersymmetries as diffeomorphisms in the fermionic directions of $\tilde{G}$. In this respect supersymmetry transformations have a geometric interpretation similar to the one in the superfield approach.

The supervielbein field $\sigma^{A}$ is a 1 -superform living in $\tilde{G}$. The coordinates of $\tilde{G}$ are the spacetime coordinates $x^{\mu}$, corresponding typically to the translation subgroup of $G$, Grassmann coordinates $\theta^{\alpha}$, corresponding to the fermionic generators of $G$, and other coordinates corresponding to gauge directions. Diffeomorphisms in these last coordinates produce gauge transformations, and the dependence of the fields on these coordinates can be removed via a finite gauge transformation. At the end of the game all fields depend on $x$ and $\theta$.

Still one has a great redundancy, since $\sigma^{A}$ is expanded as a superspace 1-form as

$$
\sigma^{A}(x, \theta)=\sigma^{A}(x, \theta)_{a} d x^{a}+\sigma^{A}(x, \theta)_{\alpha} d \theta^{\alpha}
$$

Typically the fields one wants to retain as dynamical fields in this formulation are given by $\sigma^{A}(x, \theta=0)$. In other words one has to eliminate the extra degrees of freedom due to the $\theta$ dependence and to the $d \theta$ components.

The variational principle involves variations of the fields, and variations of the embedding in $\tilde{G}$ of the bosonic submanifold. The resulting equations yield the usual spacetime field equations, together with the constraints needed to remove the redundant degrees of freedom ("rheonomic constraints"). 
In terms of these ingredients, the $D=3, N=1$ rheonomic action is defined as the integral over a bosonic submanifold $\mathcal{M}^{(3)}$ of the supermanifold $\mathcal{S} \mathcal{M}^{(3 \mid 2)}$ as follows

$$
S_{\text {rheo }}\left[\sigma, \mathcal{M}^{(3)}\right]=\int_{\mathcal{M}^{(3)} \subset \mathcal{S} \mathcal{M}^{(3 \mid 2)}} \mathcal{L}^{(3)}(x, \theta, d x, d \theta)
$$

and depends on the superforms $\sigma$ and on the embedding of $\mathcal{M}^{(3)}$ into the supermanifold $\mathcal{S} \mathcal{M}^{(3 \mid 2)}$. Changing the embedding corresponds to a diffeomorphism and it can be compensated by a change of the Lagrangian $\mathcal{L}^{(3)}(x, \theta, d x, d \theta)$, generated by a Lie derivative. Therefore the variational equations can be obtained by varying the fields for an arbitrary embedding, and considering the resulting equations as 2 -form equations on the whole superspace. Projections of these equations in the fermionic directions ( $d \theta$ directions) yield the rheonomic constraints, necessary to remove unwanted degrees of freedom. The correct component action is retrieved by setting $\theta=0$ and $d \theta=0$ (see the textbook [17]) .

The supersymmetry of the action is expressed as a diffeomorphism in the fermionic directions of the supermanifold and therefore the variation of $\mathcal{L}^{(3)}$ is given by

$$
\delta \mathcal{L}^{(3)}=\ell_{\epsilon} \mathcal{L}^{(3)}=d\left(\iota_{\epsilon} \mathcal{L}^{(3)}\right)+\iota_{\epsilon} d \mathcal{L}^{(3)} .
$$

If the Lagrangian satisfies

$$
\iota_{\epsilon} d \mathcal{L}^{(3)}=0
$$

the variation of $\mathcal{L}^{(3)}$ is a total derivative and the action is invariant. Condition (2.9) is in fact equivalent to the rheonomic constraints mentioned above.

The form of $\mathcal{L}^{(3)}$ has a direct correspondence with the component action, to which it reduces after setting $\theta=0$ and $d \theta=0$. It is less compact than the superfield formulation, but more transparently related to the component action.

We have argued that the local symmetries of the group manifold action are the diffeomorphisms on the supergroup manifold. This certainly holds true if one considers a group manifold action resulting from the integration of a top form on $\tilde{G}$. Since the past literature on group manifold actions for supergravity makes little reference to superintegration theory, this point has needed some clarification, reported in [12,20], and involves Poincaré duals and integral top forms.

\subsection{Equivalence}

The component action obtained in the two formulations must be the same or, at least, related by field redefinitions. Therefore there must exist a mother action which interpolates between the two formulations. This action is the rheonomic action. The way to integrate a 3-form on a submanifold of a bigger manifold is by constructing a Poincaré dual of that submanifold, and denoting it by $\mathbb{Y}^{(0 \mid 2)}$ the supersymmetric action is given by

$$
S_{\text {susy }}[\phi]=\int_{\mathcal{S M}^{(3 \mid 2)}} \mathcal{L}^{(3 \mid 0)} \wedge \mathbb{Y}^{(0 \mid 2)}
$$

where $\mathcal{L}^{(3 \mid 0)}$ is the rheonomic Lagrangian used in (2.7) and the integration is on the complete supermanifold. The Poincaré dual (also known as PCO) localizes the full supermanifold to the submanifold. Integration on supermanifolds is discussed in several papers (see 
for example [12] for the definition of the Poincaré dual on supermanifolds). Only the integral forms can be integrated. The complex of differential forms on a supermanifold contains the pseudo-forms which are polynomials in $d x^{a}, d \theta^{\alpha}, \delta^{(p)}\left(d \theta^{\alpha}\right)$ (where $\delta^{(r)}$ are $r$-derivatives of the delta function). They are characterized by two numbers $(p \mid q)$ : the form degree $p$ and the picture number $q$ where the latter counts the number of delta functions. In general $(p \mid q)$-forms are integral forms on $\mathcal{S} \mathcal{M}^{(p \mid q)}$, and can be integrated on this supermanifold. The integral forms of $\mathcal{S} \mathcal{M}^{(3 \mid 2)}$ are those with $(3 \mid 2)$ and they can be integrated on $\mathcal{S M}^{(3 \mid 2)}$. Thus the Lagrangian $\mathcal{L}^{(3 \mid 0)} \wedge \mathbb{Y}^{(0 \mid 2)}$ is an integral form, built as the product of the rheonomic action $\mathcal{L}^{(3 \mid 0)}$, which is a $(3 \mid 0)$-superform (constructed as discussed above), and the Poincaré dual/PCO $\mathbb{Y}^{(0 \mid 2)}$, which is a $(0 \mid 2)$-form.

The Poincaré dual/PCO $\mathbb{Y}^{(0 \mid 2)}$ is closed and not exact (it belongs to the cohomology class $\left.H^{(0 \mid 2)}\left(d, \mathcal{S} \mathcal{M}^{(3 \mid 2)}\right)\right)$, and its variation under the change of the embedding of $\mathcal{M}^{(3)}$ into $\mathcal{S} \mathcal{M}^{(3 \mid 2)}$ is $d$-exact:

$$
\delta \mathbb{Y}^{(0 \mid 2)}=d \Omega^{(-1 \mid 2)}
$$

where $\Omega^{(-1 \mid 2)}$ is an integral form with negative form degree (derivatives of the delta functions act as negative degree forms: for example $d \theta \delta^{\prime}(d \theta)=-\delta(d \theta)$ ). Then, any variation of the embedding is ineffective if $\mathcal{L}^{(3 \mid 0)}$ is closed (the action does not depend on the embedding). Also, if two $\mathbb{Y}$ 's are related by $d$-exact terms, namely if they belong to the same cohomology class, the corresponding actions are equivalent.

We propose the two different choices

$$
\mathbb{Y}_{\mathrm{st}}^{(0 \mid 2)}=\theta^{2} \delta(d \theta), \quad \quad \mathbb{Y}_{\text {susy }}^{(0 \mid 2)}=V^{a} \wedge V^{b} \gamma_{a b}^{\alpha \beta} \iota_{\alpha} \iota_{\beta} \delta^{2}(\psi),
$$

where $\left(V^{a}, \psi^{\alpha}\right)$ are the components of the supervielbein $E^{A} . \iota_{\alpha}$ is the derivative of the delta function with respect to its argument and $\delta^{2}(\psi)=\epsilon_{\alpha \beta} \delta\left(\psi^{\alpha}\right) \wedge \delta\left(\psi^{\beta}\right)$. Inserting the first PCO $\mathbb{Y}_{\mathrm{st}}^{(0 \mid 2)}$ we project the Lagrangian to $\mathcal{L}^{(3 \mid 0)}(x, 0, d x, 0)$ yielding the component action. The second choice leads to the superspace action in (2.2). The main goal of the present work is to prove this equivalence.

In a related work [21], the equivalence of the different formulations of $N=1$ super Chern-Simons theory has been studied. The flat version of $\mathbb{Y}_{\text {susy }}^{(0 \mid 2)}$ is discussed and its properties are described in that paper.

\section{$3 \quad D=3, N=1$ supergravity in the two frameworks}

The theory contains a vielbein 1 -form $V^{a}$ with 3 off-shell degrees of freedom $(d(d-1) / 2$ in $d$ dimensions), and a gravitino $\psi^{\alpha}$ with 4 off-shell degrees of freedom $\left((d-1) 2^{[d / 2]}\right.$ in $d$ dimensions for Majorana or Weyl). The mismatch can be cured by an extra bosonic d.o.f., here provided by a bosonic 2-form auxiliary field $B$. As recalled, the group-geometric procedure to build supergravity actions starts from a superalgebra. In the case at hand the superalgebra is the superPoincare algebra, generated by $P_{a}, L_{a b}$ and $Q_{\alpha}$ (the translation generators, the Lorentz generators and the supersymmetry charges). The structure constants of the superalgebra are encoded in the Cartan-Maurer equations

$$
d \sigma^{A}+\frac{1}{2} C_{B C}^{A} \sigma^{B} \wedge \sigma^{C}=0
$$


where the left-invariant one-forms $\sigma^{A}$ are a cotangent (vielbein) basis, dual to the tangent vectors on the supergroup manifold $G$. In the present case the cotangent basis is given by the vielbein $V^{a}$, the spin connection $\omega^{a b}$ and the gravitino $\psi^{\alpha}$. The algebra is further extended with a 2 -form $B$ in order to match the degrees of freedom (and thus becomes a Free Differential Algebra (FDA), see for ex. [17]).

The generalized Cartan-Maurer equations of the FDA yield the definitions of the Lorentz curvature, the torsion, the gravitino field strength and the 2-form field strength:

$$
\begin{aligned}
R^{a b} & =d \omega^{a b}-\omega^{a}{ }_{c} \omega^{c b} \\
R^{a} & =d V^{a}-\omega^{a}{ }_{b} V^{b}-\frac{i}{2} \bar{\psi} \gamma^{a} \psi \equiv \mathcal{D} V^{a}-\frac{i}{2} \bar{\psi} \gamma^{a} \psi \\
\rho & =d \psi-\frac{1}{4} \omega^{a b} \gamma_{a b} \psi \equiv \mathcal{D} \psi \\
H & =d B-\frac{i}{2} \bar{\psi} \gamma^{a} \psi V^{a}
\end{aligned}
$$

where $\mathcal{D}$ is the Lorentz covariant derivative, and exterior products between forms are understood. The Cartan-Maurer equations are invariant under rescalings

$$
\omega^{a b} \rightarrow \lambda^{0} \omega^{a b}, V^{a} \rightarrow \lambda V^{a}, \psi \rightarrow \lambda^{\frac{1}{2}} \psi, B \rightarrow \lambda^{2} B
$$

Taking exterior derivatives of both sides yields the Bianchi identities:

$$
\begin{aligned}
\mathcal{D} R^{a b} & =0 \\
\mathcal{D} R^{a}+R_{b}^{a} V^{b}-i \bar{\psi} \gamma^{a} \rho & =0 \\
\mathcal{D} \rho+\frac{1}{4} R^{a b} \gamma_{a b} \psi & =0 \\
d H-i \bar{\psi} \gamma^{a} \rho V^{a}+\frac{i}{2} \bar{\psi} \gamma^{a} \psi R^{a} & =0
\end{aligned}
$$

invariant under the rescalings (3.6).

As explained above, the redundancy introduced by promoting each physical field to a superfield has to be tamed by imposing some algebraic constraints on the curvature parametrizations. They are known as conventional constraints in the superspace language and as rheonomic parametrizations in the group-manifold approach. We use the following parametrizations

$$
\begin{aligned}
R^{a b} & =R^{a b}{ }_{c d} V^{c} V^{d}+\bar{\theta}_{c}^{a b}{ }_{c} \psi V^{c}+c_{1} f \bar{\psi} \gamma^{a b} \psi \\
R^{a} & =0 \\
\rho & =\rho_{a b} V^{a} V^{b}+c_{2} f \gamma_{a} \psi V^{a} \\
H & =f V^{a} V^{b} V^{c} \epsilon_{a b c} \\
d f & =\partial_{a} f V^{a}+\bar{\psi} \Xi
\end{aligned}
$$

with

$$
\bar{\theta}_{c, \alpha}^{a b}=c_{3}\left(\bar{\rho}_{c}{ }^{[a} \gamma^{b]}\right)_{\alpha}+c_{4}\left(\bar{\rho}^{a b} \gamma_{c}\right)_{\alpha} \quad \Xi_{\alpha}=c_{5} \epsilon^{a b c}\left(\gamma_{a} \rho_{b c}\right)_{\alpha}
$$


The coefficients $c_{1}, c_{2}, c_{3}, c_{4}, c_{5}$ are fixed by the Bianchi identities to the values:

$$
c_{1}=\frac{3 i}{2}, c_{2}=\frac{3}{2}, c_{3}=2 i, c_{4}=-i, c_{5}=-\frac{i}{3 !}
$$

The $V V V$ component $f$ of $H$ scales as $f \rightarrow \lambda^{-1} f$, and is identified with the auxiliary scalar superfield of the superspace approach of ref [10]. Note that, thanks to the presence of the auxiliary field, the Bianchi identities do not imply equations of motion for the spacetime components of the curvatures. To compare with the superspace approach and the superspace action, we have to clarify the role of the superfield $f$.

The superspace formulation of supergravity in $D=3$ follows a different path, and considers the supervielbein $E^{A}$ and the spin connection $\omega_{B}^{A}$ as fundamental fields, with $\mathrm{A}=\mathrm{a}, \alpha$. The index of the supervielbein now runs only on the superspace directions, and $E^{A}$ contains the fields of the rheonomic approach as $E^{a}=V^{a}, E^{\alpha}=\psi^{\alpha}$.

Again there is a huge redundancy in that formulation, and one has to impose some constraints. First, one imposes the soldering constraint on the spin connection

$$
\omega_{B}^{A}=\left(\begin{array}{cc}
\omega_{b}^{a} & 0 \\
0 & \frac{1}{4}\left(\gamma^{a b}\right)_{\beta}^{\alpha} \omega_{a b}
\end{array}\right),
$$

where the off-diagonal pieces are set to zero and the spinorial part of the connection is related to the Lorentz spin connection. As a consequence the supercurvature

$$
R^{A B}=d \omega^{A B}-\omega_{C}^{A} \wedge \omega^{C B}
$$

has nonvanishing components $R^{a b}, R^{\alpha \beta}=\frac{1}{4}\left(\gamma_{a b}\right)_{\beta}^{\alpha} R^{a b}$ with

$$
R^{a b}=R_{r s}^{a b} E^{r} \wedge E^{s}+R_{r \sigma}^{a b} E^{r} \wedge E^{\sigma}+R_{\rho \sigma}^{a b} E^{\rho} \wedge E^{\sigma},
$$

The superfields $R_{r s}^{a b}, R_{r \sigma}^{a b}$ and $R_{\rho \sigma}^{a b}$ correspond to the analogous terms in (3.11). Similarly, one considers the supertorsion

$$
T^{A}=d E^{A}-\omega_{B}^{A} \wedge E^{B}
$$

which has the following expansion on the supervielbein basis

$$
\begin{aligned}
& T^{a}=T_{r s}^{a} E^{r} \wedge E^{s}+T_{r \sigma}^{a} E^{r} \wedge E^{\sigma}+T_{\rho \sigma}^{a} E^{\rho} \wedge E^{\sigma}, \\
& T^{\alpha}=T_{r s}^{\alpha} E^{r} \wedge E^{s}+T_{r \sigma}^{\alpha} E^{r} \wedge E^{\sigma}+T_{\rho \sigma}^{\alpha} E^{\rho} \wedge E^{\sigma} .
\end{aligned}
$$

To reduce the independent components, one imposes the conventional constraints

$$
T_{\rho \sigma}^{a}=\frac{1}{2} i \gamma_{\rho \sigma}^{a}, \quad T_{r \sigma}^{a}=0, \quad T_{\rho \sigma}^{\alpha}=0, \quad T_{r \sigma}^{\alpha}=2 i\left(\gamma_{r}\right)_{\sigma}^{\alpha} R,
$$

The Bianchi identities then imply $R_{\rho \sigma}^{a b}=0$ and $T_{r s}^{a}=\epsilon_{r s}^{a} R$, where $R$ is a superfield containing the scalar auxiliary field as first component, the gravitino curvature as mixed component, and the Ricci scalar as $\theta^{2}$ component. The solution for the other components can be found in $[10,22]$. The supertorsion $T^{a}$ differs from $R^{a}$ defined in (3.3) by a term bilinear in fermions, and this reflects into the first constraint given above. 
Using these constraints, one finds that the only independent off-shell degrees of freedom (vielbein, gravitino and scalar auxiliary field) are contained in the components $E_{\mu}^{\alpha}$ and $E_{m}^{\alpha}$ of the superform expansion $E^{\alpha}=E_{\mu}^{\alpha} d \theta^{\mu}+E_{m}^{\alpha} d x^{m}$. Using the gauge symmetries, one can identify the physical and auxiliary fields.

Comparing the analysis in the superspace and the analysis in the rheonomic approach, we find that the auxiliary superfield $f$ has to be identified with $R$. Indeed we observe that, by a change of the spin connection, one can set to zero the last term in the parametrization of the curvature $R^{a b}$ in (3.11), namely $R_{\rho \sigma}^{a b}=0$. This change in the spin connection produces a change of $R^{a}$ in (3.12) such that $R_{r s}^{a}=\epsilon_{r s}^{a} f$. Comparing with the constraint $T_{r s}^{a}=\epsilon_{r s}^{a} R$ of the superfield approach one finds $f=R$.

\section{The actions and their equivalence}

To uncover the relation between the superspace action (2.2), the rheonomic action (2.7) and the component action, we have to discuss them in the corresponding frameworks.

With the usual group-geometrical methods, the action is determined as in (2.7) and the Lagrangian $\mathcal{L}^{(3)}$ reads

$$
\mathcal{L}^{(3)}=R^{a b} V^{c} \epsilon_{a b c}+2 i \bar{\psi} \rho+\alpha\left(f H-\frac{1}{2} f^{2} V^{a} V^{b} V^{c} \epsilon_{a b c}\right)
$$

This action is obtained by taking for the Lagrangian $\mathcal{L}^{(3)}$ the most general Lorentz scalar 3 -form, given in terms of the curvatures and 1-form fields (cotangent basis of $\tilde{G}$ ), invariant under the rescalings discussed above, and then requiring that the variational equations admit the vanishing curvatures solution

$$
R^{a b}=R^{a}=\rho=H=f=0,
$$

and also imply the constraints, arising from the $\delta \omega^{a b}$ and $\delta f$ variations:

$$
R^{a}=0, \quad H=f \epsilon_{a b c} V^{a} V^{b} V^{c} .
$$

The remaining parameter $\alpha$ is fixed by requiring the closure of $\mathcal{L}^{(3)}$, i.e. $d \mathcal{L}^{(3)}=0$. This yields $\alpha=6$, and ensures the off-shell closure of the supersymmetry transformations given below. The action is invariant under off-shell supersymmetry transformations which are easily computed by taking the Lie derivative of the fields along the fermionic directions (tangent vectors dual to $\psi^{\alpha}$ ):

$$
\begin{aligned}
\delta_{\varepsilon} V^{a} & =-i \bar{\psi} \gamma^{a} \varepsilon \\
\delta_{\varepsilon} \psi & =\mathcal{D} \varepsilon \\
\delta \omega^{a b} & =\bar{\theta}^{a b}{ }_{c} \varepsilon V^{c}-3 i f \bar{\psi} \gamma^{a b} \varepsilon \\
\delta_{\varepsilon} B & =-i \bar{\psi} \gamma^{a} \varepsilon V^{a} \\
\delta_{\varepsilon} f & =0
\end{aligned}
$$

and close on all the fields without need of imposing the field equations. 
Varying $\omega^{a b}, V^{a}, \psi, B$ and $f$ leads to the equations of motion:

$$
\begin{aligned}
R^{a} & =0 \\
R^{a b} & =9 f^{2} V^{a} V^{b}+\frac{3 i}{2} f \bar{\psi} \gamma^{a b} \psi \\
\rho & =\frac{3}{2} \gamma_{a} \psi V^{a} \\
d f & =0 \\
H & =f V^{a} V^{b} V^{c} \epsilon_{a b c}
\end{aligned}
$$

Notice that the equations of motion are obtained from the rheonomic action principle (as explained in the textbook [17]), by varying the action keeping the submanifold fixed. They are 2-form equations and can be expanded on the basis $V^{a}, \psi^{\alpha}$.

Let us move to the superspace action. As we have seen in the previous section, after imposing the constraints we are left with a superfield $R$ which contains the auxiliary field, the Ricci scalar and the Rarita-Schwinger term. To build the action we therefore consider the expression

$$
\mathcal{F}(x, \theta)=R \operatorname{Sdet}(E)
$$

where $\operatorname{Sdet}(E)$ is the superdeterminant of the supervielbein $E^{A}$. The expression in $\mathcal{F}(x, \theta)$ is a superfield and transforms as discussed in section 2. By expanding at the second order in $\theta$ 's, one can retrieve the component action. However, the computation is rather cumbersome already in the present simplified context. A better way to derive the component action from (4.14) is the use of the ectoplasmic integration theory [23-26]. We refer to $[1,10]$ for a complete discussion and for the equations of motion in superspace.

Finally, we are ready to discuss the relation between the two actions. As explained in the introduction, the mother theory interpolating between the rheonomic action, the superspace action and the component action is described by the superintegral:

$$
S_{\mathrm{SG}}=\int_{\mathcal{S M}^{(3 \mid 2)}} \mathcal{L}^{(3 \mid 0)} \wedge \mathbb{Y}^{(0 \mid 2)}
$$

where the Lagrangian $\mathcal{L}^{(3 \mid 0)}$ is the rheonomic action given in (4.1). It is a (3|0)-form and it is closed because of the parametrizations (3.11)-(3.15). ${ }^{3}$ The choice of the Poincaré dual/PCO $\mathbb{Y}^{(0 \mid 2)}$ allows us to interpolate between the component action and the superspace action.

To retrieve the usual spacetime action one chooses for the Poincaré dual/PCO the following (0|2)-form:

$$
\mathbb{Y}_{\mathrm{st}}^{(0 \mid 2)}=\epsilon_{\alpha \beta} \theta^{\alpha} \theta^{\beta} \epsilon_{\gamma \delta} \delta\left(d \theta^{\gamma}\right) \delta\left(d \theta^{\delta}\right)
$$

It is closed and not exact, and it is an element of the cohomology $H^{(0 \mid 2)}\left(d, \mathcal{S M}^{(3 \mid 2)}\right)$. The integration over the $d \theta$ 's is performed by integrating on the Dirac delta functions,

\footnotetext{
${ }^{3}$ The dependence of the fields on the gauge (Lorentz) coordinates factorizes, and reduces to a multiplicative factor in front of the integral over the superspace.
} 
that imposes $d \theta=0$. Berezin integration in (4.15) yields an ordinary spacetime action, integrated on $\mathcal{M}^{(3)}$ :

$$
S_{\mathrm{SG}}=\int_{\mathcal{M}^{(3)}} \mathcal{L}^{(3 \mid 0)}(\theta=0, d \theta=0)
$$

where all forms depend only on $x$ because of the two $\theta$ 's in $\mathbb{Y}_{\mathrm{st}}^{(0 \mid 2)}$. Notice that the supersymmetry variation of $\mathbb{Y}_{\mathrm{st}}^{(0 \mid 2)}$ is not zero, but is exact, and therefore the integrand is supersymmetric only up to a total derivative.

The action (4.15) depends in general on the choice of the bosonic $M^{3}$ submanifold. This choice is encoded in the Poincaré dual/PCO $\mathbb{Y}_{\mathrm{st}}^{(0 \mid 2)}$. Varying the submanifold via a diffeomorphism in the $\theta$ directions corresponds to a variation of $\mathbb{Y}_{\mathrm{st}}^{(0 \mid 2)}$ given by an exact form, since the Lie derivative $\mathcal{L}_{\epsilon}=i_{\epsilon} d+d i_{\epsilon}$ applied on $\mathbb{Y}_{\mathrm{st}}^{(0 \mid 2)}$ yields $d\left(i_{\epsilon} \mathbb{Y}_{\mathrm{st}}^{(0 \mid 2)}\right)$. Then the variation of the action due to the variation of the submanifold is:

$$
\delta S_{\mathrm{SG}}=\int_{\mathcal{S M}^{3 \mid 2}} \mathcal{L}^{(3 \mid 0)} \wedge d\left(i_{\epsilon} \mathbb{Y}_{\mathrm{st}}^{(0 \mid 2)}\right)
$$

Integrating by parts and noting that $0=i_{\epsilon}\left(d \mathcal{L}^{(3 \mid 0)} \wedge \mathbb{Y}_{\mathrm{st}}^{(0 \mid 2)}\right)$ since $d \mathcal{L}^{(3 \mid 0)} \wedge \mathbb{Y}_{\mathrm{st}}^{(0 \mid 2)}=0$ (because it exceeds the maximal rank of an integral form), we find that $\delta S_{\mathrm{SG}}=0$ if

$$
i_{\epsilon} d \mathcal{L}^{(3 \mid 0)}=0
$$

Another Poincaré dual can be chosen as follows

$$
\mathbb{Y}_{\text {susy }}^{(0 \mid 2)}=V^{a} V^{b} \gamma_{a b}^{\alpha \beta} i_{\alpha} i_{\beta} \delta^{2}(\psi)
$$

with

$$
i_{\alpha} \equiv \frac{\delta}{\delta \psi^{\alpha}}, \quad \delta^{2}(\psi) \equiv \epsilon_{\gamma \delta} \delta\left(\psi^{\gamma}\right) \delta\left(\psi^{\delta}\right)
$$

We prove in the appendix that $\mathbb{Y}_{\text {susy }}^{(0 \mid 2)}$ is connected to the Poincare dual/PCO in (4.16) by a $\theta$-diffeomorphism. Therefore their difference is exact (since a Lie derivative acting on a closed form gives an exact form), and we find the equivalence:

$$
S_{\mathrm{SG}}=\int_{\mathcal{S M}^{(3 \mid 2)}} \mathcal{L}^{(3 \mid 0)} \wedge \mathbb{Y}_{\mathrm{st}}^{(0 \mid 2)}=\int_{\mathcal{S M}^{(3 \mid 2)}} \mathcal{L}^{(3 \mid 0)} \wedge \mathbb{Y}_{\text {susy }}^{(0 \mid 2)}
$$

since $d \mathcal{L}^{(3 \mid 0)}=0$. The choice of $\mathbb{Y}_{\text {susy }}^{(0 \mid 2)}$ is also dictated by Hodge duality: indeed it is the Hodge dual of the $(3 \mid 0)$-form:

$$
\bar{\psi} \gamma_{a} \psi V^{a}
$$

which is closed (by the 3d Fierz identity) and not exact. Since Hodge duality maps $(3 \mid 0)$ cohomology classes into (0|2)-cohomology classes [27] we know a priori that $\mathbb{Y}_{\text {susy }}^{(0 \mid 2)}$ is closed and not exact, and fulfills the requirements for a Poincaré dual.

Computing now the term with $\mathbb{Y}_{\text {susy }}^{0 \mid 2}$, we see that only the first two terms of $\mathcal{L}^{(3 \mid 0)}$ contribute, and using the curvature parametrizations for $R^{a b}$ and $\rho$ one finds:

$$
S_{\mathrm{SG}}=6 i \int_{\mathcal{M}^{(3 \mid 2)}} f \epsilon_{a b c} V^{a} V^{b} V^{c} \delta^{2}(\psi)=6 i \int\left[d^{3} x d^{2} \theta\right] f \operatorname{Sdet}(E)
$$


where $E=\left(V^{a}, \psi^{\alpha}\right)$ is the supervielbein in superspace and we have used

$$
\mathrm{Vol}^{(3 \mid 2)}=\epsilon_{a b c} V^{a} \wedge V^{b} \wedge V^{c} \wedge \delta^{2}(\psi)=\operatorname{Sdet}(E) d^{3} x \delta^{2}(d \theta)
$$

Recalling that $f$ is identified with the scalar superfield $R$ we finally conclude that the two actions are indeed equivalent. ${ }^{4}$

The present formulation permits also the introduction of a cosmological constant term. This is achieved by shifting the superfield $f$ by a constant term $f \rightarrow f+\sqrt{\Lambda}$ or equivalently, in the superspace framework, by shifting the superfield $R$. The result is that the action acquires a new term proportional to the volume form $\mathrm{Vol}^{(3 \mid 2)}$. It is interesting to notice that this new term

$$
\Omega^{(3 \mid 2)}=\left(\sqrt{\Lambda} \bar{E} \gamma_{a} E E^{a}-6 i \Lambda \epsilon_{a b c} E^{a} E^{b} E^{c}\right),
$$

is closed using the rheonomic parametrizations (3.11)-(3.15).

In conclusion, the group-manifold rheonomic Lagrangian $\mathcal{L}^{(3 \mid 0)}$, integrated on superspace, yields both the usual spacetime $D=3$ and $N=1$ supergravity action, and its superspace version. The essential ingredients of the proof are Poincaré duals differing by a total derivative, and the rheonomic constraints with the auxiliary field that ensure $d \mathcal{L}^{(3 \mid 0)}=0$.

\section{Outlook and perspectives}

With the present work, we have established a precise mathematical relation between two different superspace formulations of supergravity. We have used the $N=1, D=3$ supergravity for simplicity. Nonetheless, the present formulation is applicable to any supergravity model and in particular to $N=1,2,4, \ldots D=4$ supergravity and higher dimensional models. The mathematical framework permits to explore different choices of PCO interpolating different superspace formulations. An important remark: the equivalence between the different formulations holds because the Lagrangian $\mathcal{L}^{(3 \mid 0)}$ is closed, and this is a consequence of the existence of the auxiliary fields for the model at hand, i.e. the existence of an off-shell formulation of the theory. This agrees with the common belief about the existence of an action principle in superspace. Note however that the rheonomic formulation of supergravity models (such as for example $D=11$ and $D=10 N=2$ supergravities) is available even in absence of auxiliary fields and it would certainly be interesting to test the present analysis on such models.

As a final comment we observe that the form $\mathcal{L}^{(3 \mid 0)} \wedge \mathbb{Y}^{(0 \mid 2)}$ is integrable on the supermanifold $\mathcal{S} \mathcal{M}^{(3 \mid 2)}$, but is definitely not the only one. One can wonder whether it would be possible to construct a supergravity action as a non-factorized (3|2) integral form

$$
S_{\mathrm{SG}}=\int_{\mathcal{S M}^{(3 \mid 2)}} \mathcal{L}^{(3 \mid 2)}
$$

where $\mathcal{L}^{(3 \mid 2)}=\sum_{l=0}^{2} \mathcal{L}^{(3 \mid l)} \wedge \mathbb{Y}^{(0 \mid 2-l)}$. Indeed, it can be shown that in $N=1 D=3$ case, there exists such a possibility and it will be discussed separately.

\footnotetext{
${ }^{4}$ The relation between integral forms and superspace formulation has also been used to formulate massive supergravity in the multivielbein formulation in [28].
} 


\section{Acknowledgments}

We would like to thank C. Maccaferri, D. Francia, F. Del Monte, P. Fré and M. Porrati for useful discussions and remarks.

\section{A Properties of the susy PCO}

Closure. The closure of $\mathbb{Y}_{\text {susy }}^{(0 \mid 2)}$ may be inferred by Hodge duality with the cohomology class $\bar{\psi} \gamma_{a} \psi V^{a}$. In this appendix we prove it directly. We use here the superspace notations for the supervielbein $E^{a}=V^{a}, E^{\alpha}=\psi^{\alpha}$. We check that, by using the conventional supergravity constraints, the PCO

$$
\mathbb{Y}^{(0 \mid 2)}=E^{a} \wedge E^{b}\left(\gamma_{a b}\right)^{\alpha \beta} \iota_{\alpha} \iota_{\beta} \delta^{2}(E)
$$

is closed and not exact where $\delta^{2}(E) \equiv \epsilon_{\rho \sigma} \delta\left(E^{\rho}\right) \delta\left(E^{\sigma}\right)$.

It is invariant under Lorentz symmetry since all tangent indices are contracted with Lorentz invariant tensors. It is also closed. To prove it, we observe

$$
\begin{aligned}
d\left(E^{a}\right. & \left.\wedge E^{b}\left(\gamma_{a b}\right)^{\alpha \beta} \iota_{\alpha} \iota_{\beta}\left(\epsilon_{\rho \sigma} \delta\left(E^{\rho}\right) \delta\left(E^{\sigma}\right)\right)\right) \\
= & 2\left(T^{a} \wedge E^{b}\left(\gamma_{a b}\right)^{\alpha \beta} \iota_{\alpha} \iota_{\beta}\left(\epsilon_{\rho \sigma} \delta\left(E^{\rho}\right) \delta\left(E^{\sigma}\right)\right)\right) \\
& +2\left(E^{a} \wedge E^{b}\left(\gamma_{a b}\right)^{\alpha \beta} \iota_{\alpha} \iota_{\beta}\left(\epsilon_{\rho \sigma} \iota_{\gamma} \delta\left(E^{\rho}\right) \wedge T^{\gamma} \wedge \delta\left(E^{\sigma}\right)\right)\right)
\end{aligned}
$$

We expand the torsion $T^{A}$ in the vielbein basis: $T^{A}=T_{B C}^{A} E^{B} \wedge E^{C}$ and we obtain for the first term:

$$
\begin{aligned}
T^{a} \wedge & E^{b}\left(\gamma_{a b}\right)^{\alpha \beta} \iota_{\alpha} \iota_{\beta}\left(\epsilon_{\rho \sigma} \delta\left(E^{\rho}\right) \delta\left(E^{\sigma}\right)\right) \\
& =\left(T_{c d}^{a} E^{c} \wedge E^{d}+T_{\delta \gamma}^{a} E^{\delta} \wedge E^{\gamma}\right) \wedge E^{b}\left(\gamma_{a b}\right)^{\alpha \beta} \iota_{\alpha} \iota_{\beta}\left(\epsilon_{\rho \sigma} \delta\left(E^{\rho}\right) \delta\left(E^{\sigma}\right)\right) \\
& =\left(R \epsilon_{c d}^{a} E^{c} \wedge E^{d}+2 i \gamma_{\delta \gamma}^{a} E^{\delta} \wedge E^{\gamma}\right) \wedge E^{b}\left(\gamma_{a b}\right)^{\alpha \beta} \iota_{\alpha} \iota_{\beta}\left(\epsilon_{\rho \sigma} \delta\left(E^{\rho}\right) \delta\left(E^{\sigma}\right)\right)
\end{aligned}
$$

where we have used the parametrization of the torsion. Due to antisymmetrization, we can recast the first term as follows

$$
R \epsilon_{c d}^{a} \epsilon^{c d b} E^{3}\left(\gamma_{a b}\right)^{\alpha \beta} \iota_{\alpha} \iota_{\beta}\left(\epsilon_{\rho \sigma} \delta\left(E^{\rho}\right) \delta\left(E^{\sigma}\right)\right)=0
$$

where $E^{3}=\frac{1}{3 !} \epsilon_{a b c} E^{a} \wedge E^{b} \wedge E^{c}, \epsilon_{c d}^{a} \epsilon^{c d b}=\eta^{a b}$, and the term vanishes because of the antisymmetry of $\gamma_{a b}$. The second term in (A.3) can be written as

$$
2 i \gamma_{\delta \gamma}^{a}\left(\gamma_{a b}\right)^{\alpha \beta} \iota_{\alpha} E^{\delta} \wedge \iota_{\beta} E^{\gamma} \wedge E^{b} \epsilon_{\rho \sigma} \delta\left(E^{\rho}\right) \delta\left(E^{\sigma}\right)=0
$$

where we have used $\iota_{\alpha} E^{\beta}=\delta_{\alpha}^{\beta}$ by definition.

Let study the second piece in (A.2)

$$
\begin{aligned}
E^{a} \wedge & E^{b}\left(\gamma_{a b}\right)^{\alpha \beta} \iota_{\alpha} \iota_{\beta}\left[\epsilon_{\rho \sigma} \iota_{\gamma} \delta\left(E^{\rho}\right) \wedge\left(T_{c d}^{\gamma} E^{c} \wedge E^{d}+T_{c \delta}^{\gamma} E^{c} \wedge E^{\delta}\right) \wedge \delta\left(E^{\sigma}\right)\right] \\
& =E^{a} \wedge E^{b} \wedge E^{c} T_{c \delta}^{\gamma}\left(\gamma_{a b}\right)^{\alpha \beta} \epsilon_{\rho \sigma} \iota_{\alpha} \iota_{\beta}\left[\iota_{\gamma} \delta\left(E^{\rho}\right) \wedge E^{\delta} \wedge \delta\left(E^{\sigma}\right)\right] \\
& =\epsilon^{a b c} T_{c \delta}^{\gamma}\left(\gamma_{a b}\right)^{\alpha \beta} \epsilon_{\rho \sigma} \iota_{\alpha} \iota_{\beta}\left[\iota_{\gamma} \delta\left(E^{\rho}\right) \wedge E^{\delta} \wedge \delta\left(E^{\sigma}\right)\right] E^{3} \\
& =R \epsilon^{a b c}\left(\gamma_{c}\right)_{\delta}^{\gamma}\left(\gamma_{a b}\right)^{\alpha \beta} \epsilon_{\rho \sigma} \iota_{\alpha} \iota_{\beta}\left[\iota_{\gamma} \delta\left(E^{\rho}\right) \wedge E^{\delta} \wedge \delta\left(E^{\sigma}\right)\right] E^{3}=0
\end{aligned}
$$


where we have used $T_{c \delta}^{\gamma}=R\left(\gamma^{c}\right)_{\delta}^{\gamma}$, the fact that $\iota_{\alpha} \iota_{\beta} \iota_{\gamma}$ is totally symmetric with respect to the spinorial indices, and the Fierz identity in $D=3$.

Relation between $\mathbb{Y}_{\mathbf{s t}}^{(\mathbf{0} \mid 2)}$ and $\mathbb{Y}_{\text {susy }}^{(\mathbf{0} \mid \mathbf{2})}$. There are two ways to compute the difference between $\mathbb{Y}_{\text {st }}^{(0 \mid 2)}$ and $\mathbb{Y}_{\text {susy }}^{(0 \mid 2)}$. The first uses the fact that they are, from the mathematical point of view, the Poincaré duals of embeddings of a submanifold $\mathcal{M}^{(3)}$ into $\mathcal{S M}^{(3 \mid 2)}$. Therefore, if the two embeddings gives two submanifolds in the same homology class the corresponding Poincaré duals belongs to the same cohomology class. Thus, the difference is $d$-exact. The second way to verify this is to observe that the variation under a diffeomorphism $\xi$ (in the supermanifold) of the PCO is $d$-exact

$$
\delta \mathbb{Y}^{(0 \mid 2)}=\mathcal{L}_{\xi} \mathbb{Y}^{(0 \mid 2)}=d\left(\iota_{\xi} \mathbb{Y}^{(0 \mid 2)}\right)
$$

Therefore, we can relate two PCO's by infinitesimal changes of the background. With that we can relate $\mathbb{Y}_{\text {susy }}^{(0 \mid 2)}$ with the flat one

$$
\mathbb{Y}_{\text {susy } / \text { flat }}^{(0 \mid 2)}=V^{a} \wedge V^{b}\left(\gamma_{a b}\right)^{\alpha \beta} \iota_{\alpha} \iota_{\beta} \delta^{2}(\psi)
$$

where $V^{a}=d x^{\alpha}+\frac{1}{2} i \bar{\theta} \gamma^{a} d \theta$ and $\psi^{\alpha}=d \theta^{\alpha}$.

The flat Cartan-Maurer equations immediately imply that $d V^{a}=\frac{i}{2} d \bar{\theta} \gamma^{a} d \theta, d \psi=0$ and therefore

$$
\begin{aligned}
d \mathbb{Y}_{s s / f l a t}^{(0 \mid 2)} & =i \bar{\psi} \gamma^{a} \psi \wedge V^{b}\left(\gamma_{a b}\right)^{\alpha \beta} \iota_{\alpha} \iota_{\beta} \delta^{2}(\psi) \\
& =i \iota_{\alpha} \iota_{\beta}\left(\bar{\psi} \gamma^{a} \psi\right) \wedge V^{b}\left(\gamma_{a b}\right)^{\alpha \beta} \delta^{2}(\psi) \\
& =2 i \gamma_{\alpha \beta}^{a}\left(\gamma_{a b}\right)^{\alpha \beta} V^{b} \delta^{2}(\psi)=0
\end{aligned}
$$

It is manifestly invariant under supersymmetry, and satisfies an interesting equation. In $D=3$, with $N=1$ we have the following Chevalley-Eilenberg cohomology class representative

$$
\omega^{(3 \mid 0)}=\bar{\psi} \gamma_{a} \psi V^{a}
$$

which is supersymmetric (it is written in terms of supersymmetric variables) and is closed: $d \omega^{(3 \mid 0)}=0$ by using the Fierz identities. Now we can construct a $(3 \mid 2)$ form as follows

$$
\begin{aligned}
\mathbb{Y}_{s s / f l a t}^{(0 \mid 2)} \wedge \omega^{(3 \mid 0)} & =V^{a} \wedge V^{b}\left(\gamma_{a b}\right)^{\alpha \beta} \iota_{\alpha} \iota_{\beta} \delta^{2}(\psi) \wedge V^{c} \bar{\psi} \gamma_{c} \psi \\
& =\epsilon_{a b c} V^{a} \wedge V^{b} \wedge V^{c} \epsilon_{\alpha \beta} \delta\left(\psi^{\alpha}\right) \delta\left(\psi^{\beta}\right)=\mathrm{Vol}^{(3 \mid 2)}
\end{aligned}
$$

which is the volume form of the supermanifold $\mathcal{S} \mathcal{M}^{(3 \mid 2)}$. In this sense, the PCO $\mathbb{Y}_{\text {susy }}^{(0 \mid 2)}$ flat is the Hodge dual to the Chevalley-Eilenberg cohomology class (A.9). Expanding the flat bosonic vielbeins $V^{a}$ and using the derivative on the Dirac delta functions, we can rewrite $\mathbb{Y}_{\text {susy } / \text { flat }}^{(0 \mid 2)}$ as follows

$$
\mathbb{Y}_{\text {susy } / f l a t}^{(0 \mid 2)}=d\left[\Omega^{(-1 \mid 2)}\right]+\mathbb{Y}_{\mathrm{st}}^{(0 \mid 2)}
$$


where $\Omega^{(-1 \mid 2)}$ is a $(-1 \mid 2)$ form in the space of integral forms. This proves that the difference between the supersymmetric flat PCO and the spacetime PCO is an exact term. The difference between flat and curved supersymmetric PCO's is again $d$-exact (since it is produced via a diffeomorphism), so that $\mathbb{Y}_{\text {st }}^{(0 \mid 2)}$ and $\mathbb{Y}_{\text {susy }}^{(0 \mid 2)}$ indeed belong to the same cohomology class.

Open Access. This article is distributed under the terms of the Creative Commons Attribution License (CC-BY 4.0), which permits any use, distribution and reproduction in any medium, provided the original author(s) and source are credited.

\section{References}

[1] S.J. Gates Jr., M.T. Grisaru, M. Roček and W. Siegel, Superspace or one thousand and one lessons in supersymmetry, Front. Phys. 58 (1983) 1 [hep-th/0108200] [INSPIRE].

[2] P. van Nieuwenhuizen, $D=3$ conformal supergravity and Chern-Simons terms, Phys. Rev. D 32 (1985) 872 [INSPIRE].

[3] B.M. Zupnik and D.G. Pak, Superfield formulation of the simplest three-dimensional gauge theories and conformal supergravities, Theor. Math. Phys. 77 (1988) 1070 [Teor. Mat. Fiz. 77 (1988) 97] [INSPIRE].

[4] P.S. Howe and R.W. Tucker, A locally supersymmetric and reparametrization invariant action for a spinning membrane, J. Phys. A 10 (1977) L155 [INSPIRE].

[5] P.S. Howe and R.W. Tucker, Local supersymmetry in $(2+1)$-Dimensions. 1. Supergravity and differential forms, J. Math. Phys. 19 (1978) 869 [INSPIRE].

[6] P.S. Howe and R.W. Tucker, Local supersymmetry in $(2+1)$-dimensions. 2. An action for a spinning membrane, J. Math. Phys. 19 (1978) 981 [inSPIRE].

[7] T. Uematsu, Structure of $N=1$ conformal and Poincaré supergravity in $(1+1)$-dimensions and $(2+1)$-dimensions, Z. Phys. C 29 (1985) 143 [InSPIRE].

[8] T. Uematsu, Constraints and actions in two-dimensional and three-dimensional $N=1$ conformal supergravity, Z. Phys. C 32 (1986) 33 [INSPIRE].

[9] M. Brown and S.J. Gates Jr., Superspace Bianchi identities and the supercovariant derivative, Annals Phys. 122 (1979) 443 [INSPIRE].

[10] F. Ruiz Ruiz and P. van Nieuwenhuizen, Lectures on supersymmetry and supergravity in $(2+1)$-dimensions and regularization of supersymmetric gauge theories, in the proceedings od the $2^{\text {nd }} M e x i c a n$ School, Recent developments in gravitation and mathematical physics, December 1-7, Tlaxcala, Mexico (1996).

[11] M. Becker et al., $M$ theory on $\operatorname{Spin}(7)$ manifolds, fluxes and $3 D, N=1$ supergravity, Nucl. Phys. B 683 (2004) 67 [hep-th/0312040] [InSPIRE].

[12] L. Castellani, R. Catenacci and P.A. Grassi, The geometry of supermanifolds and new supersymmetric actions, Nucl. Phys. B 899 (2015) 112 [arXiv:1503. 07886] [InSPIRE].

[13] T. Voronov and A. Zorich, Integration on vector bundles, Funct. Anal. Appl. 22 (1988) 94.

[14] R. Catenacci, M. Debernardi, P.A. Grassi and D. Matessi, Balanced superprojective varieties, J. Geom. Phys. 59 (2009) 1363 [arXiv:0707.4246] [InSPIRE]. 
[15] R. Catenacci, M. Debernardi, P.A. Grassi and D. Matessi, Cech and de Rham Cohomology of Integral Forms, J. Geom. Phys. 62 (2012) 890 [arXiv: 1003.2506] [InSPIRE].

[16] E. Witten, Notes on supermanifolds and integration, arXiv:1209.2199 [INSPIRE].

[17] L. Castellani, R. D'Auria and P. Fré, Supergravity and superstrings: a geometric perspective, World Scientific, Singapore (1991).

[18] L. Castellani, P. Fré and P. van Nieuwenhuizen, A review of the group manifold approach and its application to conformal supergravity, Annals Phys. 136 (1981) 398 [INSPIRE].

[19] L. Castellani, Group geometric methods in supergravity and superstring theories, Int. J. Mod. Phys. A 7 (1992) 1583 [inSPIRE].

[20] L. Castellani, R. Catenacci and P.A. Grassi, Supergravity actions with integral forms, Nucl. Phys. B 889 (2014) 419 [arXiv: 1409.0192] [INSPIRE].

[21] P.A. Grassi and C. Maccaferri, Chern-Simons theory on supermanifolds, JHEP 09 (2016) 170 [arXiv: 1606.06609] [INSPIRE].

[22] S.M. Kuzenko, U. Lindström and G. Tartaglino-Mazzucchelli, Off-shell supergravity-matter couplings in three dimensions, JHEP 03 (2011) 120 [arXiv:1101.4013] [INSPIRE].

[23] S.J. Gates, Jr., Ectoplasm has no topology: the prelude, in Supersymmetries and quantum symmetries, J. Weiss A.E. Ivanov, Springer, Germany (1999), hep-th/9709104 [INSPIRE].

[24] S.J. Gates Jr., Ectoplasm has no topology, Nucl. Phys. B 541 (1999) 615 [hep-th/9809056] [INSPIRE].

[25] S.J. Gates, Jr. and G. Tartaglino-Mazzucchelli, Ectoplasm and superspace integration measure for $2 D$ supergravity with four spinorial supercurrents, J. Phys. A 43 (2010) 095401 [arXiv: 0907.5264] [INSPIRE].

[26] S.M. Kuzenko, U. Lindström, M. Roček, I. Sachs and G. Tartaglino-Mazzucchelli, Three-dimensional $\mathcal{N}=2$ supergravity theories: from superspace to components, Phys. Rev. D 89 (2014) 085028 [arXiv:1312.4267] [INSPIRE].

[27] L. Castellani, R. Catenacci and P.A. Grassi, Hodge dualities on supermanifolds, Nucl. Phys. B 899 (2015) 570 [arXiv: 1507.01421] [InSPIRE].

[28] F. Del Monte, D. Francia and P.A. Grassi, Multimetric supergravities, JHEP 09 (2016) 064 [arXiv: 1605.06793] [INSPIRE]. 